\title{
Algunas reflexiones sobre las diferencias de género en las conductas infractoras de niñas y adolescentes en Chile (Reflections about gender differences in girls' delinquency behaviors in Chile)
}

OÑATI SOCIO-LEGAL SERIES VOLUME 10, ISSUE 1S (2020), 218S-239S: THE FOURTH WAVE OF

FEMINISM: FROM SOCIAL NETWORKING AND SELF-DETERMINATION TO SISTERHOOD

DOI LINK: HTTPS://DOI.ORG/10.35295/OSLS.IISL/0000-0000-0000-1164

RECEIVED 12 MARCH 2019, ACCEPTED 14 OCTOBER 2020

\author{
MARCEla Pilar AEDO RiverA* \\ PATRICIA VARELA PINO*
}

\section{Resumen}

En este artículo se presenta una reflexión sobre las diferencias de género en las conductas infractoras de niñas y adolescentes a la luz, principalmente, de los hallazgos de un estudio del Programa de Atención Integral Familiar (PAIF) 24 horas de la Subsecretaría de Prevención del Delito de Chile. El marco interpretativo utilizado para la presente reflexión es el de la criminología feminista y desde esta perspectiva se plantea como las niñas y adolescentes han sido históricamente relegadas en los estudios sobre la delincuencia juvenil y la necesidad de incorporar en éstos la estratificación de género en la sociedad patriarcal, especialmente de cara a identificar las diferencias de género existentes en la delincuencia femenina adolescente y su tratamiento.

\section{Palabras clave}

Adolescentes infractoras; criminología feminista; delincuencia juvenil

\section{Abstract}

This article presents a reflection on the gender differences of offending behaviors in girls and adolescents, in light - mainly - of the findings of a study of the

\footnotetext{
* Universidad de Valparaíso. Doctora en Derecho por la Universidad Autónoma de Barcelona. Investigadora del Centro de Investigaciones de Filosofía del Derecho y Derecho Penal y Profesora de criminología y sociología jurídica de la Escuela de Derecho, Universidad de Valparaíso, Chile. Dirección de email: marcela.aedo@uv.cl

* Socióloga de la Universidad de Chile, candidata a Magister en Estudios de Género por la misma Universidad y posee Diplomados en Estudios de Género y Derechos Humanos y Políticas Públicas. Cuenta con una amplia trayectoria en investigación aplicada y asesorías a actores públicos en materias de género. En los últimos años, ha participado en diversos estudios y asesorías para el sector justicia, destacando la asesoría técnica para la elaboración de la Política de Género y No Discriminación del Poder Judicial de Chile y el Diagnóstico de Género del Ministerio Público de Chile. Dirección de email: patricia.varela@gmail.com
} 
Comprehensive Family Attention Program (PAIF, for its acronym in Spanish) 24 hours of the Undersecretary of Prevention of the Crime, from Chile. The interpretive framework used for this reflection is that of feminist criminology, and from this perspective it is considered how girls and adolescents have been historically relegated in studies on juvenile delinquency, and the need to incorporate gender stratification in the patriarchal society, especially focusing to identify the existing gender differences in female adolescent delinquency and its treatment.

\section{Key words}

Girls and juvenile justice; feminist criminology; juvenile delinquency 


\section{Table of contents}

1. El Sistema de Responsabilidad Penal Adolescente en Chile y las adolescentes infractoras: algunas consideraciones sobre su invisibilización ......

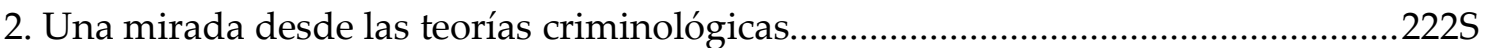

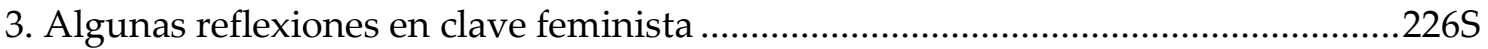

3.1. Algunas consideraciones generales .......................................................226S

3.2. La mirada a la delincuencia y criminalización de las niñas y jóvenes ............227S

4. Una primera aproximación a las diferencias de género en niñas/os y adolescentes

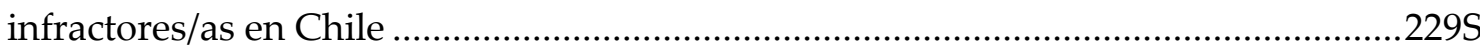

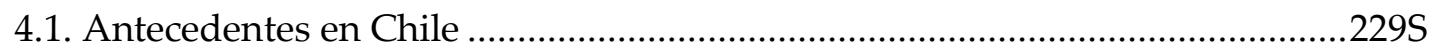

4.2. Análisis del Estudio de género y factores de riego sociodelictual en el Programa de Atención Integral Familiar (PAIF) 24 horas....................................................231S

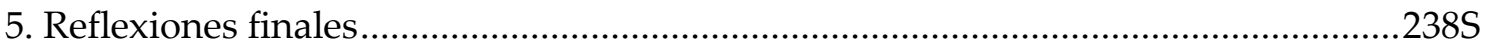

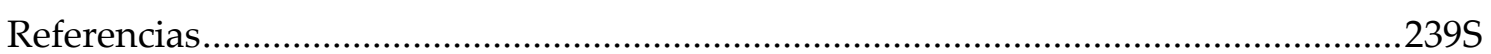


CITA DE APERTURA.

(Autor 2013, 184)

CITA DE APERTURA № 2 .

(Lee Dema, Founder of St Matthew's youth project, Brixton, London)

\section{El Sistema de Responsabilidad Penal Adolescente en Chile y las adolescentes infractoras: algunas consideraciones sobre su invisibilización}

La Ley № 20.084 que regula la responsabilidad penal adolescente (LRPA) -vigente desde el 8 de junio de 2007- introdujo en Chile un modelo de responsabilidad penal especial para los y las adolescentes mayores de 14 y menores de 18 años, cuyo objetivo primordial era dar una respuesta a la crisis del Sistema de Justicia de Menores que rigió en gran parte del siglo $\mathrm{XX}^{1}$ y adecuar la legislación a los requerimientos contenidos en la Convención sobre los Derechos del Niño (en adelante CDN).

Desde el punto de vista jurídico la LRPA no regula específicamente la situación de las adolescentes infractoras; sin embargo, su Reglamento sí lo hace, aunque sólo respecto de aquellas privadas de libertad. En el título V (Normas comunes a los centros privativos de libertad) párrafo $8^{\circ}$ denominado Normas especiales para mujeres adolescentes, comprende aspectos tales como, el derecho a un centro o sección exclusivos para la población adolescente femenina; personal especializado; derecho a la privacidad e integridad; derecho a la salud (particularmente aspectos relativos a las adolescentes embarazadas) y derecho a convivir con sus hijos/as, quienes podrán permanecer en los centros hasta la edad de dos años.

Estas normas son complementadas con las Orientaciones Técnicas del Servicio Nacional de Menores (2011) para adolescentes privados/as de libertad tanto en régimen cerrado como en internación provisoria y que hacen referencia a una intervención cognitiva conductual especial para mujeres.

Desde el punto de vista empírico, en Chile los datos desagregados por sexo son excepcionalísimos y muy generales. Por otra parte, consultados expertos/as e instituciones nacionales (Defensoría Penal Pública, Ministerio Público y Sename Servicio Nacional de Menores), hemos confirmado la casi nula existencia de estudios especializados, con contadas excepciones, como el informe elaborado por la Defensoría Penal Pública el año 2010 sobre las barreras de género en el marco de la Ley de Responsabilidad Penal Adolescente, un estudio publicado el año 2014 desarrollado por la académica Claudia Reyes Quilodrán titulado ¿Por qué las adolescentes chilenas delinquen? Y un último -que contiene información relevante- encargado por la Subsecretaría de la Prevención del Delito, Ministerio del Interior y Seguridad Pública, del Gobierno de Chile, elaborado por ISÓNOMA Consultorías Sociales (2016) y que tuvo como principal objetivo realizar un análisis y evaluación de variables de género, factores

\footnotetext{
${ }^{1}$ En América Latina se dictaron leyes de menores entre los años 1919 (Argentina) y 1939 (Venezuela). En Chile la doctrina de la "situación irregular" se materializa en la Ley 4.447 de 1928. Para una revisión de la historia del paso del paradigma tutelar al de protección integral en Chile, ver Cillero 1994.
} 
de riesgo y comportamiento infractor de los niños, niñas y adolescentes ingresados al PAIF 24 horas. $^{2}$

Transcurridos 13 años, las evaluaciones de la Ley y su implementación han sido más bien negativas, destacando ante todo la falta de información estadística y particularmente la debilitada especialidad del sistema, que se orienta -siguiendo tendencias internacionales- hacia un modelo más punitivo o de control de riesgos (Aedo y Collell 2017).

Esta precaria especialidad afecta fundamentalmente a las adolescentes mujeres, quienes -en general- se mantienen ausentes de la regulación jurídica específica (LRPA y Reglamento), de los estudios que analizan la delincuencia juvenil, de los foros de discusión y de las estadísticas oficiales: como si no existieran. De este modo, podemos concluir que el sujeto sobre el que se articula el modelo de responsabilidad nacional, en particular, el tratamiento jurídico penal y las políticas penitenciarias, es el menor varón, lo que nuevamente pone en evidencia el carácter particularmente incompleto de la ciudadanía de las niñas y adolescentes.

Asimismo, cabe tener presente que en la actualidad el sistema de responsabilidad penal adolescente carece de una profunda deslegitimación, en particular porque no cuenta con un adecuado sistema de garantías integral de la infancia, que constituye el mínimo ético para sostener, al menos discursivamente, que los niños, niñas y adolescentes son ciudadanos en los términos de la CIDN, sujetos de derechos y por tanto de obligaciones (Aedo y Collell 2017). ${ }^{3}$ Es decir, previo a la decisión político criminal de castigar a los y las adolescentes, un Estado respetuoso de su infancia, debe cumplir las exigencia de los tratados internacionales y demás normas internacionales sobre el tema -en particular la $\mathrm{CDN}-\mathrm{y}$ de este modo, aunque sea de modo indirecto, primero debe evitar la criminalización de los niños y niñas mediante la prevención de los delitos, es decir asegurando la protección de sus derechos básicos, tales como la salud y educación (Beloff 2011).

\section{Una mirada desde las teorías criminológicas}

$\mathrm{Al}$ revisar las principales teorías criminológicas que tradicionalmente han explicado el fenómeno delictivo, es posible apreciar como la mujer/niña ha sido invisibilizada o estereotipada (Smart 1976, Zaffaroni 1993).

Como ha señalado Carol Smart (1976) en muchos estudios criminológicos, la mujer no se menciona, su propia existencia se ignora o se considera tan insignificante como para tomarse en cuenta. El desviado, el criminal o el actor, siempre es masculino; siempre es su racionalidad, su motivación, su alienación o su víctima. La experiencia del mundo femenino nunca se expresa, aun cuando puede ser y frecuentemente lo es, en efecto, diferente de la experiencia masculina.

\footnotetext{
${ }^{2}$ Se trata de un programa dirigido a niños, niñas y adolescentes entre 10 y 17 años (y sus respectivas familias) con problemas conductuales asociados a transgresiones, factores de riesgo socio delictual e integración social. Cabe hacer presente que el estudio comprende a niños/as (menores de 14 años e inimputables) y adolescentes (responsables penalmente). La distinción legal entre niños/as y adolescentes descansa en la Ley de Tribunales de Familia (Artículo 16 inciso 3ㅜㅡ, Ley № 19.968) y en la Ley de Responsabilidad Penal Adolescente (№ 20.084).

3 Sobre la ciudadanía de los niños y las niñas, ver Baratta 1999.
} 
Asimismo, al hacer una mirada de las primeras teorías criminológicas, de corte positivista (Lombroso y Ferrero 1898), éstas caracterizaron a la mujer delincuente como biológicamente anormal no solamente por su conducta desviada, sino porque no sería una mujer completa. Y de ahí se desprende un doble oprobio: la condena legal por el delito y la condena social por la anormalidad biológica o sexual. De hecho, las primeras teorías concluyen que por ser una doble excepción la mujer criminal es un monstruo (Lombroso y Ferrero 1895, en Miralles 1983, 123-124).

Se afirmaba por estos autores que la mujer tendría una mayor pasividad, lo que estaría determinado fisiológicamente; por ello, serían más obedientes a la ley, cuestión que explicaría su baja participación en hechos delictivos. Al mismo tiempo, serían potencialmente amorales, es decir engañosas, frías, calculadoras, seductoras y malévolas (Van Swaaningen 1989, 85-86).

Y en el caso de las mujeres, la teoría del atavismo lombrosiano, no las conduciría a cometer delitos sino a la prostitución, lo que constituiría, para estos autores, la forma obvia de desviación femenina (Antony 2005, 22).

Los postulados positivistas expuestos influyeron en posteriores teorías que explican la delincuencia femenina. Y si bien comenzaron gradualmente a incluir otros factores, se centraron exclusivamente en la anatomía, y particularmente en su sexualidad (ChesneyLind y Shelden 2004, 101).

Así, por ejemplo, el trabajo de Sheldon y Eleonor Glueck, quienes, en su obra Five Hundred Delinquent Women (1934), ${ }^{4}$ analizan el encarcelamiento de quinientas mujeres, a quienes califican como un "grupo lamentable" -la mitad de ellas habían sido prostitutas y cuatro quintos de éstas padecían enfermedades venéreas- y consideran que un trabajo exhaustivo con ellas, incluyendo el cumplimiento de largas condenas en reformatorios y la esterilización voluntaria, constituía un avance progresivo. Asimismo, documentaron los vínculos existentes entre las adolescentes involucradas con la justicia penal juvenil y su posterior relación con la justicia penal adulta. Así, por ejemplo, observaron que la mitad de su muestra adulta había sido arrestada cuando joven, ya sea por "inmoralidad" o "tenacidad" 5 y que las mujeres "nacidas y criadas en orfanatos pobres componían un grupo común" (Chesney-Lind y Shelden 2004, 102).

Para estos autores y otros posteriores como para Cowie, Cowie y Slater, Delinquency in Girls (1968), la delincuencia femenina toma predominantemente la forma de una conducta sexual (por ejemplo, promiscuidad), lo que requiere un mayor grado de maduración que los delitos de los niños. Y en cuanto a las causas de la delincuencia femenina, sin perjuicio de explorar factores ambientales o sociales, enfatizan el determinismo biológico. En este sentido señalan que:

Las diferencias entre los sexos en la predisposición hereditaria (a la delincuencia) se podría explicar por los genes vinculados al sexo. Además, el modo de la personalidad

\footnotetext{
${ }^{4}$ Un trabajo de campo en el que hacen un seguimiento a la "carrera delictiva" de 500 mujeres que han cumplido sentencia en el Massachusetts Reformatory for Women (Framingham, Massachusetts), y que, a pesar de tratar en su mayoría sobre mujeres adultas, contiene valiosa información sobre la delincuencia juvenil femenina (dos quintos de su población eran menores de 21 años).

${ }^{5}$ N. del T. En el original aparece "stubbornness".
} 
femenina, más tímido, más carente de emprendimiento, puede protegerla contra la delincuencia. (Cowie et al. 1968, 167, citado en Smart 1977, 92)

De este modo, se trataría de un modelo explicativo que se funda en patologías individuales, al que le corresponderá una ideología orientada al control, que servirá para localizar las causas de los "problemas" en individuos específicos y que suministra el conocimiento y la comprensión pertinentes a desarrollar las tecnologías apropiadas y las políticas sociales para el control de desviación miembros. Así la teoría criminológica se convierte en un medio de proporcionar nuevas tecnologías para el control o, en su defecto, un medio de legitimar las actuales políticas, que quedan justificadas como forma de tratamiento en lugar de castigos (Smart 1977, 94).

Además, este tipo de investigaciones rara vez cuestionaron la equidad de los juzgados de familia exclusivamente preocupados con la sexualidad femenina. La mayoría asumió que la población de los juzgados reflejaba exactamente la "naturaleza" de la delincuencia femenina. Por lo tanto, el tema en estos trabajos fue como diagnosticar y tratar a los miembros de esta población y devolverlas a sus roles "propios", y de ese modo protegerles de los ambientes que pudieran atraerlas a la actividad sexual. ${ }^{6}$ Obviamente, esta aproximación tendía a negar el rol que jugaba la clase social o etnia, sin perjuicio, que ambos factores fueron usados como relevantes al momento de explicar el comportamiento delictivo masculino. Asimismo, subestimaron el rol de los agentes del sistema de justicia juvenil (policía, oficiales y jueces) en la forma en que moldean el problema de la delincuencia femenina (Chesney-Lind y Shelden 2004, 106).

Teorías sociológicas posteriores, y que han explicado con mayor o menor profundidad la delincuencia juvenil, en genera han invisibilizado a las adolescentes o han caracterizado su comportamiento desde estereotipos sexistas (Aedo 2014).

Un buen ejemplo de ello es la conocida teoría de la subcultura, uno de cuyos principales representantes es Albert Cohen quien siempre se refiere al joven o jóvenes en masculino, es decir, excluye a las chicas de su tratamiento y cuando se refiere a las chicas lo hace desde caracterizaciones de tipo sexista. En efecto, en su obra más destacada, Delinquent Boys: The Culture of the Gang (1955), el autor señala que mientras los chicos están interesados en sus logros y en ser "masculinos", las chicas están interesadas en sus relaciones afectivas con chicos, espacio en una niña/adolescente encuentra su realización como tal. Y cuando reconoce la delincuencia femenina, él la define básicamente como delincuencia sexual. Y si bien el comportamiento delictivo masculino puede ser condenado por otros en el terreno moral, tiene al menos la virtud de confirmar a los ojos de todos los involucrados, su esencia masculina. De este modo, concluye el autor, el delincuente es el macho dominante (Cohen 1955, 139-140).

Será la criminología feminista la que marcará un punto de inflexión en esta mirada, al denunciar las prácticas cotidianas que, enfatizando los valores masculinos, reprimen,

\footnotetext{
${ }^{6}$ El trabajo de Vedder y Somerville The Delinquent Girl (1970) señalaba que aproximadamente un 75\% a 85\% de los delitos cometidos por las niñas revestían el carácter de ofensas sexuales. Sin embargo, este patrón era confuso porque en la mayoría de los casos gran parte de los cargos como huir de casa, incorregibilidad e ingobernabilidad, entre otros, se incorporaban en el registro oficial. Asimismo, observaron la sobrerrepresentación de las niñas afroamericanas en situación de privación de libertad en las áreas metropolitanas, lo que da cuenta del cruce de discriminaciones múltiples (género-raza) (Chesney Lind y Shelden 2004, 104)
} 
discriminan y desvaloran las experiencias femeninas, y producen una desigualdad real entre hombre y mujeres (Antony 2005, 33). 


\section{Algunas reflexiones en clave feminista}

\subsection{Algunas consideraciones generales}

La historia del sistema penal juvenil se ha construido, por lo general, con la imagen de un niño/adolescente en masculino. La identidad de género atraviesa los espacios del sistema, en los que se reproducen los sexismos y discriminaciones que las mujeres sufren en otros ámbitos. En esos mismos espacios las especificidades que provoca la realidad sexuada y marcada por relaciones desiguales de género quedan desdibujadas y/o invisibilizadas (Bodelón 2001, 2003, 2011, Bodelón y Aedo 2015).

Los estudios socio-jurídicos y criminológicos, especialmente provenientes de la criminología feminista, ${ }^{7}$ han demostrado claramente que las mujeres criminalizadas sufren una marginación y discriminación específica (Smart 1976, Lloyd 1995, Antony 2001, 2005). Especialmente significativos resultan los estudios de las mujeres en prisión (Carlen et al. 1985, Campelli et al. 1992, Grup Dona i Presó 1995) y sobre el tratamiento de éstas ante los tribunales penales (Eaton 1986). En ambos casos, hay un elemento común: el hecho de que el sistema penal tiende a consolidar la estructura de géneros y a reproducir los elementos que provocan discriminación sexual.

La idea de que el derecho fija y reproduce las identidades de género y consolida la discriminación sexual resulta especialmente interesante aplicada al ámbito de la privación de libertad en el sistema penal juvenil.

Un caso paradigmático en términos de vulneración de derechos es la situación que viven las niñas y adolescentes privadas de libertad. Derechos tales como la salud mental, derechos sexuales y reproductivas, y derecho a vivir una vida libre de violencias (especialmente abuso físico y sexual), son especialmente afectados durante la privación de libertad, según los estudios internacionales en la materia (Shufelt y Cocozza 2006, 5, UNICEF 2011, North Carolina Office of the Juvenile Defender 2012, Reforma Penal Internacional y Asociación para la Prevención de la Tortura 2013, López 2014, 2016, Conselho Nacional de Justiça 2015, Amsellem-Mainguy et al. 2017, 2018, Diniz 2017, López-Gallego et al. 2018, Grillo y García 2020).

En relación a las teorías criminológicas que han tratado de comprender el fenómeno delictivo de las mujeres y niñas, compartimos la reflexión que ha planteado Pat Carlen:

Ninguna teoría por sí sola (feminista o no) puede explicar adecuadamente los tres rasgos sobresalientes de las mujeres que delinquen y de las mujeres encarceladas: que los delitos de las mujeres son fundamentalmente delitos típicos de la gente que carece de poder; que las mujeres encarceladas pertenecen desproporcionadamente a grupos

\footnotetext{
${ }^{7}$ La criminología feminista desde mediados de los setenta ha analizado la posición desigual de las mujeres en el derecho penal, tanto como autoras de delitos, como en cuanto víctimas de ellos (Bodelón 2003, 452). Se ha señalado -algo arbitrariamente- que la fundación de la criminología feminista puede ser fijada en el año 1976 con la publicación del libro de Carol Smart Women, Crimen and Criminology: A Feminist Critique. Aunque un grupo de trabajos anteriores había abordado algunos de los temas generales que ella planteaba, el libro de Carol Smart los unió en una crítica sistemática al tratamiento de las mujeres "delincuentes" en las corrientes principales de la criminología y al abandono de las experiencias de las mujeres como víctimas en un intento de exponer algunas orientaciones para el nuevo campo de investigación feminista (Britton 2013, 39).
} 
étnicos minoritarios; y que la mayoría de las mujeres encarceladas han vivido en la pobreza la mayor parte de sus vidas. (Carlen 1992, 53, en Larrauri 1994)

En este sentido cabe destacar el gran aporte de la(s) criminología(s) feminista(s), ${ }^{8}$ vinculadas a las teorías de la reacción social, las que han sabido mostrar cómo los procesos de definición de la desviación de las mujeres y adolescentes no son neutrales, sino que están históricamente determinados y han venido condicionados por su situación de desventaja social y por la desigual distribución de poder y de recursos que interesadamente se le han impuesto para perpetuar un orden patriarcal funcional para los intereses del capitalismo (Maqueda 2014, 16).

Y siguiendo a Maqueda, consideramos que una teoría crítica feminista requiere desnaturalizar el rol social atribuido tradicionalmente a la mujer y redefinir su relación con la delincuencia. No hay nada de natural en lo femenino que justifique su separación de lo público, ni la perversidad, ni la inmadurez, ni los déficits de inteligencia o de autonomía que los dictados de la biología o de la psicología le asignaban. Se trata de una construcción cultural que tiene que ver con la estratificación sexual que opera el género en tanto sistema normativo instalado en las instituciones históricas -responsables de la socialización de hombres y mujeres-, y, por tanto, en las estructuras objetivas y subjetivas de nuestras sociedades patriarcales (Maqueda 2014, 17).

Asimismo, cabe tener presente que el género sirve de sustento a las relaciones de poder económicas en nuestro modelo social capitalista. La división sexual del trabajo en producción material y reproducción distribuye los espacios sociales, constituyéndose la clave explicativa de los sucesivos controles, ejercidos históricamente sobre las mujeres para garantizar su conformidad con lo privado y con los roles funcionales que le han sido asignados como "madre-esposa". La salvaguarda de la familia nuclear y de los valores androcéntricos que descansan en ella -en particular, el control de su sexualidadse convierten así en un soporte más del capitalismo (Radosh 1990, 116-117, Maqueda 2014, 17).

Es necesario para un pensamiento crítico cuestionar estas actitudes apropiadas de género que, desde ese ideal femenino estereotipado, se atribuyen a las mujeres y les dejan poco espacio para actuar con iniciativa, agencia e inteligencia. En palabras de Maqueda $(2014,18)$ : “Me refiero a esa tendencia tan presente todavía en la criminología, y fuera de ella, a conferir a las mujeres una debilidad y una falta de autonomía que refuerza su imagen de víctimas sistémicas".

\subsection{La mirada a la delincuencia y criminalización de las niñas y jóvenes}

En relación a las niñas, y para explicar su delincuencia y criminalización, es necesario comenzar a considerar la importancia de la estratificación de género en la sociedad patriarcal, sobre todo porque es tan importante en la formación de la vida cotidiana de los niños y las niñas. Estudios sobre el proceso de socialización indican que el aprendizaje de los roles de género es de central importancia para niñas y niños. La socialización, particularmente durante la infancia, comienza a desarrollar en las chicas

\footnotetext{
8 Cabe señalar que "si queremos delinear los conceptos feministas de justicia, deberemos primero admitir que después del inicio de los años sesenta se han desarrollado múltiples teorías feministas. Por ello no debiéramos hablar de feminismo como una perspectiva dentro de la criminología, sino más bien de criminologías feministas" (Van Swaaningen 1989, 89).
} 
atributos que, aunque apropiados para su sexo, no son altamente valorados en la sociedad dominante. Durante la adolescencia, la aplicación de los roles de género por los padres, el personal escolar, y otros en la vida de las jóvenes enfatiza además muchos elementos tradicionales sobre el lugar de la mujer, en particular la que debe controlarse a fin de no poner en peligro su reputación (Chesney-Lind y Shelden 2004, 156).

Las investigaciones sugieren que las normas y las expectativas sobre el género, particularmente para las niñas y jóvenes, se han mantenido en gran medida inalteradas a pesar del movimiento de mujeres. El desplome de la autoestima de las niñas está probablemente vinculado al excesivo énfasis en el atractivo físico, hábitos alimenticios poco saludables, así como a múltiples causas de discriminación, tales como pobreza, invisibilidad de un sistema educacional que discrimina por género y clase, violencias de barrio, violencias en el contexto familiar, violencia machista en la pareja, etc. En esta línea, la investigación sugiere que el aumento de la autoestima en las niñas, en realidad reduce su delincuencia. En cuanto a los distintos tipos de control de que son objeto las niñas, es posible afirmar que la supervisión puede resultar en menor delincuencia en algunas chicas, pero también puede alimentar la delincuencia de otras que se rebelan contra las restricciones tradicionales. Tal "control", cuando toma la forma de abuso psíquico o sexual, es una fuerza que puede causar la delincuencia de las niñas (ChesneyLind y Shelden 2004).

Sin embargo, es importante señalar que este tipo de control informal al interior de la familia (abuso psíquico o sexual) puede ser uno de los factores detonantes, aunque no el único. Y cabe mencionar que los mecanismos de control formal e informal se complementan y son funcionales al sistema patriarcal y capitalista.

En esta tarea de normalización están implicados distintos instrumentos disciplinarios: Unos, informales, que aseguran la interiorización de las pautas convencionales de la feminidad así construida: la familia, en primer lugar, pero también la escuela o la iglesia $\mathrm{y}$, desde luego, la ciencia con sus aserciones severamente discriminatorias para las mujeres. Otros, formales, de criminalización de las conductas disconformes (Maqueda 2014, 18).

Ambos, como afirma Baratta, son controles de género masculino desde el punto de vista simbólico. Y en ambos, las formas e instrumentos, así como el discurso o la ideología oficial del sistema (que lo legitima y esconde sus funciones latentes detrás de las funciones declaradas) reproducen la diferenciación social de las cualidades y de los valores masculinos y femeninos (Baratta 2000, 61).

En cuanto a las niñas de clase trabajadora o más bajas, las presiones de los guiones de género son particularmente agudas. Incapaces de competir en un concurso de popularidad construido en torno a los estándares de belleza de la clase media-alta, ellas no están necesariamente libres de estas imágenes de feminidad. Ellas también negocian en un terreno educacional más hostil y experimentan más violencia. Deben forjar sus propias soluciones, las que pueden involucrarlas en actividad ilegal, pero ellas a menudo se sienten avergonzadas por estas opciones y se culpan a sí mismas por no ser capaces de cumplir con los roles tradicionales de la mujer con éxito (Chesney-Lind y Shelden 2004, 157). 
Cabe tener presente que la feminización de la pobreza asegura a las mujeres una posición de subsistencia y de falta de oportunidades bajo el capitalismo. En este sentido Radosh señala que:

Los patrones del delito femenino no están relacionados con la etiología de las mujeres o su emancipación. Las mujeres cometen delitos a causa del sistema económico que controla su acceso a bienes escasos tales como el prestigio, el estatus, la independencia, el estándar de clase media de opulencia o incluso la seguridad económica. El comportamiento de las mujeres es a menudo etiquetado como criminal de acuerdo a las implicaciones morales del capitalismo, pero la criminalidad real de las mujeres refleja la diferencia de clase del sistema económico. De ahí que considere la autora que el capitalismo ha definido a las mujeres como criminales a partir de la estructura de clase. (Radosh 1990, 116)

Las investigaciones actuales sugieren que la delincuencia juvenil, masculina y femenina, es alimentada por elementos tales como la pobreza, problemas en la escuela, bajo rendimiento escolar, percepción de falta de acceso a legítimas oportunidades, deprivación sociocultural y percepción de posibilidad de arresto. Adicionalmente, en el caso de las niñas, un factor que puede ser relevante en su trayectoria es la victimización sexual. Decir que estas variables son importantes para la delincuencia de las chicas, sin embargo, no quiere decir que las teorías desarrolladas para describir la importancia de las variables en la vida de los varones adolescentes se puedan utilizar para explicar la delincuencia de las chicas sin modificación. El desafío es generar teorías sobre la delincuencia que sean sensibles al contexto patriarcal de todo comportamiento, tanto los conformes como los que infringen la ley (Chesney-Lind y Shelden 2004, 157).

\section{Una primera aproximación a las diferencias de género en niñas/os y adolescentes infractores/as en Chile}

\subsection{Antecedentes en Chile}

Tal como señala Claudia Reyes, los estudios que recogen evidencia empírica que permita detectar cuáles son los factores incidentes en la conducta delictiva de los jóvenes son escasos. Esta limitada cantidad de estudios se ve agudizada al indagar sobre investigaciones que expliquen por qué las adolescentes delinquen (Reyes 2014, 6). A pesar de que estudios señalan que en esta materia existen diferencias relevantes entre sexos (Larraín 2006, en Reyes 2014, 6).

En este contexto, un estudio pionero en la materia es el desarrollado por Claudia Reyes (2014), en el que tomó una muestra representativa de 135 jóvenes, hombres y mujeres, recluidos/as en centros de justicia juvenil de tres regiones del país, con los/as cuales realizó entrevistas en profundidad y se les aplicó el Test MACI, para posteriormente comparar los resultados por sexo.

Los resultados de dicho estudio arrojaron diferencias de género en los siguientes ámbitos:

Conducta delictiva:

- Hombres y mujeres comienzan a la misma edad promedio (12 años), aunque hay mayor porcentaje de mujeres que comienzan de manera más tardía.

- En ambos sexos el primer delito es el hurto. 
- $\quad$ El 17\% de las mujeres inició su conducta delictiva traficando drogas, mientras que ningún varón comenzó traficando.

- Ambos sexos señalan las mismas motivaciones para delinquir, siendo las primeras motivaciones el adquirir vestuario y la entretención, y la segunda motivación más recurrente, la necesidad de consumir drogas.

Porte de armas: Los varones portan más armas que las mujeres ( 8 de cada 10 contra 5 de cada 10). Si bien en ambos sexos predomina el arma de fuego, en las mujeres hay mayor porte de arma blanca.

Consumo de drogas: No hay diferencias significativas en porcentaje de hombres y mujeres que consumen, pero sí hay diferencias en el tipo de droga, pues, mientras los hombres consumen principalmente marihuana, las mujeres consumen más cocaína.

Retraso escolar: Se observó una mayor deserción escolar en mujeres que en varones (87\% en mujeres contra $76,8 \%$ en varones).

Relaciones de pareja: Los varones declaran en mayor porcentaje tener una relación de pareja (64\% contra 54\%), y además se observan las siguientes diferencias respecto a las parejas:

- Las mujeres conviven más con sus parejas que los varones (26\% contra $14 \%$ ).

- Las parejas de las mujeres son más consumidoras de drogas que las de los varones (39\% contra $8 \%$ ).

- Las parejas de las mujeres presentan más antecedentes penales que las de los varones ( $48 \%$ contra $13 \%)$.

- El 100\% de las parejas de las mujeres no trabaja ni estudia, contra un $70 \%$ de las parejas de los varones.

- Un 70\% de las parejas de las mujeres se encuentra recluida, mientras que sólo un varón declaró que su pareja se encontraba en esa situación.

Grupo de pares: ${ }^{9}$ Los varones muestran en mayor porcentaje que las mujeres participación en un grupo de pares que delinque (70\% de los hombres contra $50 \%$ de las mujeres) ${ }^{10}$.

Características del grupo familiar de origen: Las características familiares que mostraron diferencias entre hombres y mujeres fueron las siguientes:

- La orfandad, ya sea materna o paterna, es estadísticamente significativa en las mujeres.

- La conducta de consumo problemático de drogas de los padres es significativa en los hombres.

\footnotetext{
${ }^{9}$ Cabe señalar que la investigadora dejó las relaciones de pareja dentro de la dimensión "grupo de pares", sin embargo, se ha preferido separarla dadas las significativas diferencias que se evidenciaron entre hombres y mujeres.

${ }^{10}$ Estos dos últimos ítems muestran que, a diferencia de los varones, las mujeres tienden a mantener una relación de pareja afectiva con personas con conductas delictivas. Mientras que los hombres, si bien se relacionan con otras personas con conductas delictivas, lo hacen en el grupo de pares, pero sus relaciones de pareja las establecen con personas que mayoritariamente no delinquen.
} 
- La gran mayoría de los y las jóvenes vive principalmente con la madre, pero son ligeramente más las chicas que se encuentran en esta situación (47,8\% en comparación al 43,7\% de los hombres).

- El 13\% de ellas se encuentran independizadas del grupo familiar contra el $7,1 \%$ de los varones.

- La orfandad paterna y materna tiende a ser mayor en las niñas que en los niños.

Conducta de calle:

- Los hombres permanecen más en la calle y también ellos viven más en la calle.

- En promedio, comienzan a estar en la calle a los 10 años, pero la tendencia es que las mujeres lo hagan más tarde (13 años).

- Respecto a las motivaciones, los varones señalan principalmente a los amigos $(36,7 \%)$ y el evitar maltrato $(27,8 \%)$, mientras que las mujeres señalan el consumo de drogas $(42,9 \%)$ y los amigos $(35,7 \%)$.

Características de la personalidad: En relación a este ámbito, se encontraron diferencias entre hombres y mujeres en los siguientes aspectos:

- Mayor presencia de déficit atencional en los varones (37,7\% contra 17,4\%)

- Los varones presentan una mayor tendencia depresiva.

- Las mujeres presentan más intentos de suicidio.

- Si bien hombres y mujeres se infringen autolesiones en porcentajes parecidos, los varones mayoritariamente se hacen cortes (41,6\% contra el $22,7 \%$ de las mujeres) y las jóvenes realizan más intentos de suicidio (36,4\% contra el 12,9\% de los varones). La menor presencia de cortes en las mujeres se debe a que éstos no son aceptables estéticamente.

A la luz de este trabajo y de otros estudios como el de Vinet y Alarcón (2009), es posible hipotetizar que, si bien, hay factores de riesgo en los cuales la variable de género no tendría mayor incidencia, es decir, que afectarían a niñas y niños de igual manera, sí existen otros en los cuáles el género sí incidiría de manera significativa. Ejemplos de factores que se ven influenciados por el género serían la baja autoestima, la victimización sexual, la existencia de parejas con antecedentes delictivos, entre otros, todos ellos factores de riesgo que serían especialmente preponderantes en el comportamiento delictual de las niñas.

4.2. Análisis del Estudio de género y factores de riego sociodelictual en el Programa de Atención Integral Familiar (PAIF) 24 horas.

Durante el segundo semestre del año 2015 y los primeros meses del año 2016, se realizó por encargo de la Subsecretaría de Prevención del Delito, dependiente del Ministerio del Interior de Chile, el Estudio de género y factores de riego sociodelictual en el Programa de Atención Integral Familiar (PAIF) 24 horas.

El Programa de Atención Integral Familiar (PAIF) 24 horas surge en el año 2009 como una iniciativa en la cual confluyen variadas instituciones vinculadas con la seguridad pública y la infancia. El programa, nace para dar respuesta a la falta de servicios de atención psicosocial para niños, niñas y adolescentes que han tenido contacto con la 
policía, por medio del Programa de Seguridad Integrada (PSI 24 horas), por la comisión de conductas transgresoras. En relación a esto, busca abordar tres nudos considerados críticos en dicho momento: 1) falta de oferta en el territorio e invisibilización de los ingresos de los NNA provenientes del PSI 24 horas; 2) Ausencia de un modelo de gestión que apoyara el PSI 24 Horas; 3) Disparidad de criterios de implementación, del PSI, en las distintas comunas.

De este modo, surge el Programa PAIF, cuyo objetivo general es contribuir a la superación de las situaciones de vulneración y/o a la interrupción de conductas transgresoras en niños, niñas y adolescentes derivados del Programa de Seguridad Integrada PSI 24 Horas.

En el año 2014, surge la necesidad de realizar un estudio que permita evaluar si existen diferencias de género en los factores de riesgo socio-delictual en niños, niñas y adolescentes del Programa.

\subsubsection{Metodología}

El estudio fue realizado con un enfoque mixto, complementando fuentes de información del tipo cuantitativa y cualitativa con el objetivo de realizar un análisis integrado que permitiera por una parte describir y cuantificar, pero que también permitiera caracterizar en mayor profundidad fenómenos vinculados con las relaciones de género y las especificidades de niñas y adolescentes mujeres.

Para lo anterior, en un primer momento de realizó una extensa revisión bibliográfica, una revisión y sistematización de documentos internos del Programa, y revisión de experiencias internacionales en relación a la incorporación del enfoque de género en programas de intervención con niños, niñas y adolescentes infractores/as, y en programas de prevención de la delincuencia juvenil. A partir de esta revisión se construyó un marco teórico y se elaboraron dimensiones de análisis, en base a las cuales se construyeron los instrumentos cualitativos y cuantitativos.

En relación a las técnicas cuantitativas, se aplicaron 242 encuestas presenciales a niños, niñas y adolescentes participantes del Programa (de distintas comunas del país) y 155 encuestas online a equipos profesionales del Programa de todo el país. En lo que refiere a las técnicas cualitativas, se realizaron cinco entrevistas grupales a equipos PAIF, y 20 estudios de caso. Para la construcción de cada estudio de caso, se entrevistó en profundidad al niño, niña o adolescente, a su terapeuta del Programa, y a su cuidador/a principal.

Una vez que el equipo contó con toda la información cualitativa y cuantitativa para ser analizada, vale decir, con las entrevistas transcritas y las bases de datos completas y validadas, se llevaron a cabo los siguientes pasos:

$1^{\circ}$ Análisis de cada instrumento por separado. En lo que respecta a los datos cuantitativos, se realizaron análisis descriptivos, bivariados y multivariados. Por su parte, con la información cualitativa se procedió a la construcción de los casos de estudio. Para la construcción de estos últimos se empleó una pauta guía que fue construida especialmente para este fin, la cual fue complementada con una planilla Excel en la cual el equipo investigador fue registrando las citas más significativas para cada dimensión 
de análisis. Cada caso fue construido por separado, para luego realizar una conclusión del caso, de la comuna, y finalmente una conclusión general de los casos.

$2^{\circ}$ Análisis integrado. A continuación, se procedió a triangular e integrar los resultados de los análisis de los diferentes instrumentos, construyendo así un relato que integró los principales hallazgos de la investigación, a la luz del marco teórico de referencia. Los resultados fueron organizados en base a las siguientes dimensiones:

- Dimensión territorio (barrio con alta prevalencia de delitos, barrio con alta prevalencia de narcotráfico, facilidad/dificultad del acceso a drogas, y existencia de redes comunitarias/vecinales).

- Dimensión Escolar (retraso o deserción escolar, aspectos subjetivos de la escolarización, problemas escolares, bajo rendimiento, etc.).

- Dimensión Familiar (orfandad, hogares monoparentales, construcción de la figura materna, existencia de otras figuras adultas significativas, modelos de crianza, padres con historial delictual, padres con consumo de drogas, cuidadores/as con problemas de salud mental, violencia intrafamiliar, imagen negativa del grupo familiar, hacinamiento en el hogar, e inserción de la familia en redes de sociales y/o de apoyo).

- Dimensión personal (autoestima, imagen de sí mismo/a, competencias psicosociales y habilidades sociales, uso del tiempo libre, maternidad/paternidad, vulneración de derechos).

- Dimensión relaciones de pareja (convivencia, pareja que delinque, pareja con actividad -trabaja o estudia-, diferencia de edad en la pareja, violencia en la relación de pareja, y prácticas sexuales inseguras).

- Dimensión trayectoria de conductas asociales (edad del primer contacto con Carabineros, edad con la que cometió el primer delito o infracción, quién enseña o guía en la trayectoria delictual, significados atribuidos al delito).

- Dimensión de género (grado de internalización de los roles y estereotipos de género).

$3^{\circ}$ Elaboración de recomendaciones. Finalmente, el equipo elaboró una batería de recomendaciones, a partir de las cuales se buscó entregar orientaciones conceptuales y prácticas para incorporar el enfoque de género en el Programa.

\subsubsection{Principales Resultados}

\section{a) Resultados respecto a NNA}

A partir de la triangulación de los resultados obtenidos de la aplicación de las técnicas cualitativas y cuantitativas, se obtuvieron los siguientes hallazgos principales para cada dimensión de análisis:

\section{Dimensión Territorio:}

En lo que refiere al territorio, si bien se constata que este puede constituirse como un factor de riesgo para ambos sexos (en cuanto espacio de modelaje de conductas transgresoras), sí se observaron diferencias significativas en relación al género. Por un lado, quedó de manifiesto una presunta mayor accesibilidad por parte de las niñas y adolescentes mujeres a sustancias ilícitas (ellas perciben más facilidad para acceder a 
drogas que sus pares varones). Por otro lado, la relación de las niñas y jóvenes con el espacio público aparece mediatizada por prácticas de control ejercida por sus cuidadores/as en mayor medida que la de los hombres, es decir, los cuidadores y cuidadoras de niñas suelen restringir más el acceso de ellas a la calle, controlando permisos y horarios con mayor intensidad que en el caso de los niños, lo cual podría llegar a ser un factor protector para ellas. De este modo, es posible afirmar que los NNA establecen trayectorias diferenciadas según género en relación al territorio.

\section{Dimensión escolar:}

Respecto a la relación de los NNA con la institucionalidad educativa, esta aparece como problemática, siendo la deserción o la expulsión del sistema una posibilidad real para ambos sexos (un 35,3\% de la muestra -109 NNA- se encontraban fuera del sistema escolar). No obstante, las niñas y adolescentes muestran una mejor capacidad de ajuste al encuadre institucional, lo cual se podría explicar porque ellas muestran una mayor valoración del proceso escolar, así como también mayor interés e intención de reescolarizarse en el caso de aquellas que estaban fuera del sistema al momento del estudio. Un hallazgo relevante en el ámbito educativo es el hecho de que el tránsito desde la educación básica a la educación media aparece como un momento crítico para los y las jóvenes. En sus relatos se observa una relación entre esta etapa de transición y el inicio de experiencias de consumo de sustancias (legales e ilegales), la irrupción de conductas trasgresoras y/o la agudización de problemáticas conductuales, y el abandono del sistema escolar.

\section{Dimensión Familiar:}

Se observó que en las familias de los NNA del Programa son las mujeres quienes ejercen los roles de cuidado, principalmente madres y abuelas. Además, predominan los hogares donde las madres son las principales proveedoras $(48,1 \%$ contra un $24,3 \%$ en que lo es el padre), las que en su mayoría presentan trabajos muy precarios, con extensas jornadas de trabajo y muchas de ellas no terminaron sus estudios, por lo que no tienen acceso a mejores opciones de empleo.

Resultó interesante también el hecho de que las relaciones entre los jóvenes y sus cuidadores/as son diferentes según se trate de sus madres o padres. En el caso de las niñas y adolescentes, a relación entre ellas y sus aparece en un mayor grado como conflictiva, lo que no ocurre en la relación de ellas con sus padres, quienes ocupan un lugar secundario en relación a las exigencias que manifiestan las jóvenes.

La prevalencia y cristalización de los estereotipos y roles de género tradicionales en el espacio familiar aparece como un factor de riesgo sociodelictual para los NNA de ambos sexos. Las actividades de crianza y provisión ejercidas de manera estereotipada lesionan la posibilidad de una asunción de responsabilidades igualitarias y de un reconocimiento y valoración equitativos al interior del espacio familiar. En el caso de las jóvenes, la ausencia de roles alternativos para las mujeres de su entorno cercano puede devenir en la sensación de no contar con posibilidades de articular proyectos de vida atractivos, apareciendo la socialización delictual como una oferta alternativa llamativa y validada por los pares. 


\section{Dimensión personal:}

Al indagar en la autopercepción y la opinión que los NNA tienen de sí mismos/as, se observó, en especial en los casos de estudio, se apreció que, en términos generales, la opinión de sí mismo/a se constituye en un factor de riesgo, para ambos sexos, en tanto es un proceso en construcción que busca aprobación afuera, en los pares, los cuales, en los contextos en que se desenvuelven los y las jóvenes incluidas en el estudio, aparecen con algún grado de compromiso con conductas infractoras. Sin embargo, hombres y mujeres se diferencian en la forma de satisfacer necesidad de aprobación, donde las niñas la buscan en la posibilidad de tener afecto constante y los varones en la adquisición de bienes. En este punto, es importante agregar que gran parte de los NNA que forman parte del programa han sido y son muy vulnerados (vivencias de violencia, agresión sexual, negligencia parental, etc.), lo que se vincula con una baja autoestima y sensación de vulnerabilidad, la cual en muchos casos tratan de ocultar bajo la imagen de "choreza" como forma de autoafirmación, validación entre los/as pares y/o autoprotección, mecanismos que aparecen coherentes con el desarrollo de conductas delictivas.

\section{Dimensión relaciones de pareja:}

Las relaciones de pareja de los NNA pueden constituirse en factor de riesgo o factor de protección/contención dependiendo del género y las relaciones entre los sexos. En efecto, las adolescentes mujeres estarían involucradas en relaciones más complejas, caracterizadas por conductas infractoras por parte de sus parejas, la pervivencia de situaciones de control sobre sus conductas, celos, intimidación, etc., lo cual las transforma en un factor de riesgo. En el caso de los varones, sus parejas aparecen constituyéndose en factores de protección, en tanto se establecen en una variable que reorganiza el uso del tiempo libre, que cautela la vinculación con pares y aparece como un motivador para reencaminar la trayectoria vital.

Este hallazgo es coherente con el hecho que a las mujeres se las ha socializado para cuidar, proteger y contener a los otros en general y a sus parejas en particular, condición que no ha sido suficientemente incentivada en los jóvenes, y que se proyecta como una brecha en la vida adulta en lo que respecta a las tareas de cuidado en el ámbito doméstico y particularmente en la crianza de los hijos e hijas.

Dimensión trayectoria delictiva o asocial:

La mayoría de los NNA estudiados, con independencia de su sexo, ha cometido delitos (un $72,2 \%$ declara haberlos cometido). Mayoritariamente, ingresaron al Programa por delitos contra la propiedad, independientemente de su sexo. Aunque en el caso de los hombres presenta una proporción significativamente mayor. En el caso de los varones el segundo tipo de delito son las infracciones a la Ley de Drogas, a diferencia de las niñas quienes en segundo lugar ingresan por ser víctimas de vulneración de derechos. En cuanto al segundo tipo de delitos por los cuales ingresarían las adolescentes mujeres, están constituidos por las amenazas y en tercer lugar las lesiones. En relación a los delitos contra la propiedad, las niñas y adolescentes aparecieron más vinculadas a los hurtos, a diferencia de los varones que cometerían proporcionalmente más delitos de robo. En ambos sexos, se aprecian casos en que el comportamiento asocial se vincula con el consumo problemático de drogas. 
En el estudio, también se buscó indagar en las motivaciones de los NNA para la comisión de delitos y/o conductas transgresoras. En el caso de las niñas y adolescentes mujeres, existiría una motivación por satisfacer ciertas necesidades que mediante los ingresos familiares no se podrían cubrir, tales como adquirir ropa de marcas o comer en ciertos lugares normalmente inaccesibles por su precio. También se observan decisiones estratégicas de sobrevivencia como el caso de una adolescente que comete delitos para alimentar a su hijo. En el caso de los varones, se aprecia que algunas conductas delictivas están motivadas por la búsqueda constante de validación entre pares, además de la obtención de dinero para adquirir lo que deseen en el momento y la necesidad de satisfacer el consumo de marihuana.

Respecto a las trayectorias delictivas o asociales, destaca el hecho que las niñas comenzarían su trayectoria "asocial" a edades más tempranas que los varones, de hecho, mientras un 33,8\% de las niñas estudiadas tenía 14 años o menos, sólo un 18,2\% de los varones se encontraba en ese tramo. En el caso de algunos adolescentes varones, la trayectoria delictiva responde a una homosocialización delictiva entre pares en el espacio de la calle y el "espacio de diversión", situación que no se observa en la misma medida entre las mujeres, las cuales parecen estar más influenciadas por las parejas que por sus pares.

\section{Dimensión de género:}

$\mathrm{Al}$ indagar de manera directa en los roles y estereotipos de género se aprecia que los niños y adolescentes varones muestran porcentajes más cercanos a concepciones tradicionales en relación a los roles y estereotipos de género. Respecto a las motivaciones para delinquir también se apreciarían roles y estereotipos conservadores. Así, por ejemplo, ellos suelen buscar validación social y "aparecer" como "choro", imitando, en ocasiones, la actitud violenta del padre, lo que responde a un patrón de masculinidad dominante. En el caso de algunas adolescentes mujeres, se observan fuertes improntas del modelo de feminidad hegemónica y de los mandatos de género, tales como la importancia que se le da a la apariencia física -la comisión de hurtos aparece asociada a la compra ropa y enseres personales de carácter cosmético-, y el predominio de creencias propias del amor romántico en las relaciones de pareja, como la expectativa de ser parte del proceso de "sanación" de sus parejas en el caso de consumo problemático de sustancias. Este último elemento es de suma relevancia, en tanto esta idealización del amor las vuelve vulnerables a relaciones de pareja abusivas y violentas.

\section{b) Resultados respecto a los equipos profesionales del Programa}

El estudio también arrojó interesantes resultados respecto a los imaginarios de género que portan los y las profesionales que intervienen con los NNA del Programa. En este punto, es necesario relevar que los/as profesionales del Programa cuentan con un alto nivel de formación y experiencia, así como con un alto grado de compromiso con el trabajo que realizan. No obstante, no cuentan con formación en género (salvo excepciones, que corresponden a casos en los cuales por interés propio se ha cursado algún tipo de especialización en la materia), y al momento del estudio, el Programa tampoco les había ofrecido capacitación al respecto.

Los resultados de las técnicas aplicadas a profesionales muestran que estos/as tienden a caracterizar de manera genéricamente diferenciada a niños y niñas. Por un lado, a ellas 
las tienden a asociar más con la imagen de vulnerabilidad y con problemas emocionales, asimismo, en las niñas se otorga mayor valor al entorno afectivo (familia y parejas). En el caso de los niños, los asocian con características que se corresponden con el modelo de masculinidad hegemónica, tales como la temeridad, la osadía, la agresividad, que son mencionadas por varios/as de los/as terapeutas. De este modo, se observa que en la caracterización que las/os profesionales hacen de los niños y las niñas del Programa se reproducen estereotipos y roles tradicionales de género, lo cual puede estar permeado por los propios imaginarios de género de los/as profesionales y/o porque efectivamente los jóvenes del Programa presentan marcadamente los roles tradicionales de género.

Por otro lado, un porcentaje importante de los/as profesionales del Programa (40\%) cree que sí hay diferencias entre las motivaciones de NNA hombres y mujeres para delinquir. En el caso de los varones las principales motivaciones serían, desde su punto de vista, la validación frente a los pares y el obtener dinero para comprarse cosas; mientras que en las niñas las motivaciones serían las necesidades económicas y los problemas familiares. Estas percepciones de los equipos reproducen la imagen de las mujeres como un ser para otros, donde su conducta es motivada por la búsqueda del sustento económico para el bienestar familiar o bien por los problemas emocionales causados por sus relaciones afectivas. Mientras que los varones aparecen en posiciones más egoístas, preocupados de su imagen personal y social. Destaca que, en las niñas aparece en las motivaciones el "acompañar a la pareja", y que, en el caso de las niñas, las motivaciones identificadas por los/as profesionales no se condicen por completo con las relatadas por ellas mismas, las cuales sí reconocen en algunos casos intereses egoístas, tales como comprar ropa de marca, cosméticos, etc.

\section{c) Otros resultados emergentes}

Además de las dimensiones definidas a partir de la revisión bibliográfica y documental, durante el proceso de producción y análisis de información emergieron otros temas relevantes en relación al género. Uno de estos temas es la precocidad en el consumo de drogas (se considera precocidad el consumo antes de los 15 años de edad), la cual parece ser mayor en las niñas y adolescentes mujeres, puesto que el $73 \%$ de las niñas encuestadas presentan precocidad en comparación al 63\% de los varones.

Otro hallazgo a destacar es que el embarazo en muchas ocasiones es reconocido como un factor protector por parte del entorno de las adolescentes (cuidadores/as y/o terapeutas), ya que la familia se activa, la protege y ella deja el consumo en la mayoría de los casos. No obstante, este reconocimiento esconde problemas y reviste riesgos, puesto que en muchos casos un embarazo adolescente puede esconder una sexualidad desprotegida, y/o reproducir estereotipos e historias de vida presentes en el grupo familiar, que contribuyen a continuar en el círculo vicioso de la pobreza existente y exponer a la niña/adolescente a una situación de vulneración de derechos, tales como la dificultad de estudiar o de sacar adelante su proyecto vital. En otras palabras, si bien el embarazo de las jóvenes puede operar como un factor protector en el corto plazo, constituye una potencial causa de limitaciones y vulneraciones para el largo plazo. Esto se constata en el hecho de que la condición de maternidad aparece como causal de deserción escolar en un 10\% de las mujeres encuestadas. Esta cifra constituye una señal de alarma, frente a la posibilidad que estas jóvenes reproduzcan con sus hijos/as, la 
trayectoria de sus familias de origen, tanto en la reproducción de circuitos de pobreza como en la generación de trayectorias de vida desadaptativas.

Respecto a la sexualidad de los NNA, destaca el hecho que la sexualidad aparece como un ámbito problemático de las adolescentes mujeres y no de los varones, aun cuando ambos declaran mantener relaciones sexuales sin protección. Así, por ejemplo, en la encuesta a equipos, aparece como algo específico en las niñas la referencia a su sexualidad, caracterizándolas en algunos casos como "adultizadas" y "sexualizadas". A diferencia de la mirada que tienen en relación a los niños respecto de los que no se hace referencia a su sexualidad, pero sí se menciona su "adultización", pero esta vez asociada a que asumen en sus hogares el rol de proveedores.

En relación a la sexualidad, en algunos de los casos estudiados, esta se encontraba relacionada con el consumo problemático de alcohol y/o drogas. Hubo relatos en los cuales las niñas señalan haber tenido relaciones sexuales en momentos en que ellas eran incapaces de dar su consentimiento activo (por estar bajo efecto de drogas y/o alcohol), no obstante, para ellas aparecía como algo normalizado. Esta situación, sumada al hecho de que muchas veces en los grupos de pares existe presencia de hombres varones mayores de edad, puede estar dando cuenta de delitos sexuales en contra de las menores.

\subsubsection{Conclusión}

Luego de analizar los hallazgos recientemente expuestos a la luz de los antecedentes bibliográficos, es posible concluir que sí existen factores de riesgo en los cuales la variable de género incide de manera significativa, es decir, factores de riesgo que serían especialmente preponderantes en el comportamiento delictual de las niñas, o en otros casos de los niños.

\section{Reflexiones finales}

De acuerdo a información publicada recientemente por UNICEF (UNICEF et al. 2020), se observa un aumento en la participación de las mujeres en imputados/as adolescentes, ya que mientras en el año 2008 representaban un 14\%, para el año 2018 su representación había aumentado a un 22,4\%. Asimismo, si se compara la tasa de ingreso cada $100 \mathrm{mil}$ habitantes de adolescentes mujeres entre 14 y 17 años del 2008 con el 2018, se constata que han aumentado en $1,6 \%$, en oposición a lo que ocurre con los imputados adolescentes hombres que han disminuido en un $43 \%$.

Si a lo anterior se adiciona que, a partir de la revisión bibliográfica y los resultados del estudio anteriormente expuestos, es posible afirmar la existencia de factores de riesgo asociados al género, así como también motivaciones y comportamientos delictuales diferenciados en hombres y mujeres adolescentes, aparece no sólo necesario, sino también urgente, seguir investigando y profundizando en el comportamiento delictual adolescente desde una perspectiva de género, más aún ante la posibilidad de que los resultados del Sistema Penal Adolescente no están siendo los mismos para hombres y mujeres. Asimismo, otra arista necesaria de investigar y analizar dice relación con el acceso a la justicia de las adolescentes, puesto que, si ya existe abundante evidencia sobre una mayor dificultad para las mujeres para acceder a la justicia, no sería extraño que las barreras se agudicen cuando se trata de mujeres adolescentes. 


\section{Referencias}

Aedo, M., 2014. Las adolescentes y el sistema penal: Cuando la invisibilización tiene género. Tesis doctoral. Doctorado en Derecho Público y Filosofía Jurídica política. Facultad de Derecho. Universidad Autónoma de Barcelona. No publicada.

Aedo, M., y Collell, A., 2017. Las adolescentes infractoras y el sistema de responsabilidad penal adolescente en Chile: El problema de las pocas olvidadas. En: B. Amaral Machado y A. Pereira, eds., Justiça juvenil: Paradigmas e experiências comparadas. Sao Paulo: Marcial Pons.

Amsellem-Mainguy, Y., Coquard, B., y Vuattoux, A. 2017. Mes vrais potes, ils sont dehors. L'adolescence en prisión. Revue des politiques sociales et familiales [en línea], $\mathrm{n}^{\circ} 125,57-69$. Disponible en: https://www.persee.fr/doc/caf 243145012017 num 12513243 [Con acceso el 25 de noviembre de 2020].

Amsellem-Mainguy, Y., Coquard, B., y Vuattoux, A., 2018. Normes sociales, sexuelles et genrées des jeunes détenus en France au moment de leur passage à l'âge adulte. Revue Jeunes et Societe [en línea], 3(1). Disponible en:

http://rjs.inrs.ca/index.php/rjs/article/view/130 [Con acceso el 25 de noviembre de 2020].

Antony, C., 2001. Perspectivas de la criminología feminista en el siglo XXI. Revista de Derecho, Criminología y Ciencias Penales [en línea], 3, 249-257. Disponible en: https://dialnet.unirioja.es/servlet/articulo?codigo $=6466693$ [Con acceso el 25 de noviembre de 2020].

Antony, C., 2005. Estudio sobre violencia de género: Mujeres transgresoras. Instituto de la Mujer de la Universidad de Panamá.

Baratta, A., 1999. Infancia y democracia. En: E. García Méndez y M. Beloff, eds., Infancia, ley y democracia: Análisis crítico del panorama legislativo en el marco de la Convención Internacional sobre los Derechos del Niño. Santa Fe de Bogotá/Buenos Aires: Temis/Depalma, 31-68.

Baratta, A., 2000. El paradigma del género: De la cuestión criminal a la cuestión humana. En: H. Birgin, ed., Las trampas del poder punitivo: El género del derecho penal. Buenos Aires: Biblos, 19-37.

Beloff, M., 2011. La traducción latinoamericana de la Convención sobre Derechos del Niño. En: L. Flah, ed., Los desafíos del derecho de familia en el siglo XXI: Derechos humanos, bioética, relaciones familiares, problemáticas infanto-juveniles. Homenaje a la Dra. Nelly Minyersky. Buenos Aires: Errepar, 17-38.

Bodelón, E., 2001. La relevancia de un análisis de género en el ámbito de la justicia minoril. En: S. Rivera, ed., Pasado y presente de la justicia penal juvenil. San Salvador: Coediciones Universidad de Barcelona, UNICEF y otros, 101-113.

Bodelón, E., 2003. Género y sistema penal: los derechos de las mujeres en el sistema penal. En: R. Bergalli, ed., Sistema penal y problemas sociales. Valencia: Tirant lo Blanch Alternativa, 451-486.

Bodelón, E., 2011. Prólogo. En: CELS, Ministerio Público de la Defensa de la Nación y Procuración Penitenciaria de la Nación, eds., Mujeres en prisión: Los alcances del 
castigo [en línea]. Buenos Aires: Siglo XXI. Disponible en: https://www.cels.org.ar/web/wp-content/uploads/2011/04/Mujeres-en-prision.pdf [Con acceso el 25 de noviembre de 2020].

Bodelón, E., y Aedo, M., 2015. Las niñas en el Sistema de Justicia Penal. Anales de la Cátedra Francisco Suárez, vol. 49, 219-236.

Britton, D., 2013. Feminism in Criminology: Engendering the Outlaw. En: M. ChesneyLind y L. Pasko, eds., Girls, Women and Crime: Selected Readings. Los Ángeles: Sage, 39-51.

Campelli, E., et al., eds., 1992. Donne in carcere, Milán: Feltrinelli.

Carlen, P., et al., eds., 1985. Criminal Women: Autobiographical Accounts. Cambridge: Polity Press.

Chesney-Lind, M., y Shelden, R.G., 2004. Girls, Delinquency, and Juvenile Justice. Belmont/Londres: West/Wadsworth.

Cillero, M., 1994. Evolución Histórica de la Consideración Jurídica de la Infancia y Adolescencia en Chile. En: F. Pilotti, ed., Infancia en Riesgo Social y Políticas Sociales en Chile. Desarrollo y perspectivas del Servicio Nacional de Menores y su relación con las políticas sociales, la sociedad civil y el marco jurídico. Montevideo: Instituto Interamericano del Niño, 75-138.

Cohen, A., 1955. Delinquent Boys: The Culture of the Gang. Nueva York: Free Press.

Conselho Nacional de Justiça, 2015. Dos espaços aos direitos: a realidade na ressocialização na aplicação da medida socioeducativa de internação das adolescentes do sexo feminino em conflito com a lei nas cinco regiões do país [en línea]. Brasilia: Conselho Nacional de Justiça. Disponible en: https://carceraria.org.br/wpcontent/uploads/2018/01/formacao-cnj-pesquisa-adolesentes-fem.pdf [Con acceso el 17 de abril de 2019].

Diniz, D., 2017. Meninas fora de lei: A medida socioeducativa de internacao no Distrito Federal [en línea]. Estudio realizado para Anis Instituto de Bioética. Brasilia. Disponible en: http://anis.org.br/wp-content/uploads/2017/09/Anis-Meninas-fora-da-lei-20172.pdf [Con acceso el 25 de noviembre de 2020].

Eaton, M., 1986. Justice for Women? Family, Court and Social Control, Milton Keynes: Open University Press.

Glueck, S., y Glueck, E., 1934. Five Hundred Delinquent Women. Nueva York: A.A. Knopf.

Grillo, N.E., y García, C.C., 2020. Fuerza para subir, coraje en el descenso: un estudio sobre la resistencia de las niñas en medida socioeducativa de internamiento en Brasil. Oñati Socio-Legal Series [en línea], 10(2), 332-362. Disponible en: https://doi.org/10.35295/osls.iisl/0000-0000-0000-1046 [Con acceso el 25 de noviembre de 2020].

Grup Dona i Presó, ed., 1995. Presó i Dona. Barcelona: Grup Dona i Presó.

Larrauri, E., 1994. Control informal: las penas de las mujeres. En: E. Larrauri, ed., Mujeres, Derecho penal y criminología. Madrid: Siglo XXI de España, 1-13. 
Lloyd, A., (con H. Kennedy, pról.), 1995. Doubly Deviant, Doubly Dammed. Society's Treatment of Violent Women. Harmondsworth: Penguin.

Lombroso, C., y Ferrero, W., 1898. The Female Offender. Nueva York: D. Appleton and Company.

López Gallego, L., 2014. Proceso de reflexividad en un contexto de privación de libertad de adolescentes mujeres. Psicologia \& Sociedade [en línea], 26(3), 603-612.

Disponible en: https://doi.org/10.1590/S0102-71822014000300009 [Con acceso el 25 de noviembre de 2020].

López Gallego, L., 2016. Tramas narrativas: Adolescentes mujeres privadas de libertad en el Sistema Penal Juvenil Uruguayo. Montevideo: CSIC-UDELAR.

López-Gallego, L., Galeotti-Galmes, R., y Montes-Maldonado, C., 2018. Adolescentes mujeres y medidas no privativas de libertad: Narrativas de una experiencia etnográfica. Psicoperspectivas [en línea], 17(2). Disponible en: https://doi.org/10.5027/psicoperspectivas-Vol17-Issue2-fulltext-1212 [Con acceso el 25 de noviembre de 2020].

Maqueda, M.L., 2014. El peso del género y otras identidades culturales en la criminalización de las mujeres. Transjus Working Papers, ํㅡㄴ 4, 1-34.

Miralles, T., 1983. La mujer: el control informal. En: R. Bergalli y J. Bustos, eds., El pensamiento criminológico, estado y control (vol. 2). Bogotá: Temis, 121-148.

North Carolina Office of the Juvenile Defender, 2012. Representing Girls in the Juvenile Justice System. Informe. Agosto.

Radosh, P.F., 1990. Women and Crime in the United States: A Marxian Explanation. Sociological Spectrum [en línea], 10(1), 105-131. Disponible en: https://doi.org/10.1080/02732173.1990.9981914 [Con acceso el 25 de noviembre de 2020].

Reforma Penal Internacional y Asociación para la Prevención de la Tortura, 2013. Mujeres privadas de libertad: Una guía para el monitoreo con perspectiva de género [en línea]. Londres: Reforma Penal Internacional/Ginebra: Asociación para la Prevención de la Tortura. Disponible en: https://tortureprevention.ch/content/files res/women-in-detention-es.pdf [Con acceso el 25 de noviembre de 2020].

Reyes, C., 2014. ¿Por qué las adolescentes chilenas delinquen? Política criminal [en línea], 9(17), 1-26. Disponible en: https://doi.org/10.4067/S0718$\underline{33992014000100001}$ [Con acceso el 25 de noviembre de 2020].

Shufelt, J., y Cocozza, J., 2006. Youth with Mental Health Disorders in the Juvenile Justice System: Results from a Multi-State Prevalence Study [en línea]. Delmar: National Health Center for Mental Health and Juvenile Justice. Disponible en: http://www.unicef.org/tdad/usmentalhealthprevalence06(3).pdf [Con acceso el 25 de noviembre de 2020].

Smart, C., 1976. Women, Crime and Criminology: A Feminist Critique. Boston: Routledge \& Kegan Paul. 
Smart, C., 1977. Criminological theory: Its ideology and implications concerning women. British Journal of Sociology [en línea], 28(1), 89-100. Disponible en: https://doi.org/10.2307/589710 [Con acceso el 25 de noviembre de 2020].

UNICEF, 2011. Estado mundial de la infancia 2011. La adolescencia. Una época de oportunidades [en línea]. Nueva York: UNICEF. Disponible en:

https://www.unicef.es/sites/unicef.es/files/EMI2011.pdf [Con acceso el 25 de noviembre de 2020].

UNICEF, CIDENI y Defensoría Penal Pública, 2020. Análisis del Sistema de Responsabilidad Penal Adolescente. Serie Reflexiones Infancia y Adolescencia [en línea], no 23. Santiago de Chile: UNICEF. Disponible en:

https://www.unicef.org/chile/media/3901/file/LRPA.pdf [Con acceso el 25 de noviembre de 2020].

Van Swaaningen, R., 1989. Feminismo, criminología y derecho penal: una relación controvertida. Papers d'Estudis i Formació, no 5, 85-106.

Vinet, E., y Alarcón Bañares, P., 2009. Caracterización de personalidad de mujeres adolescentes infractoras de ley: un estudio comparativo. Paidéia (Ribeirão Preto) [en línea], 19(43), 143-152. Disponible en: https://doi.org/10.1590/S0103$\underline{863 X 2009000200002}$ [Con acceso el 25 de noviembre de 2020].

Zaffaroni, E.R., 1993. La mujer y el poder punitivo. En: R. Camacho y A. Facio, eds., Sobre patriarcas, jerarcas, patrones y otros varones. Una mirada género-sensitiva de derecho. San José: ILANUD, 89-99. 